SUPPLEMENT

\title{
Value of neuropsychological testing after head injuries in football
}

\author{
P McCrory, M Makdissi, G Davis, A Collie
}

See end of article for

authors' affiliations

Br J Sports Med 2005;39(Suppl I):i58-i63. doi: 10.1136/bjsm.2005.020776

.....................

Correspondence to: Dr P McCrory, University of Melbourne, Victoria, Australia; paulmccr@ bigpond.net.au
This paper reviews the pros and cons of the traditional paper and pencil and the newer computerised neuropsychological tests in the management of sports concussion. The differences between diagnosing concussion on the field and neuropsychological assessment at follow up and decision making with regard to return to play are described. The authors also discuss the issues involved in interpreting the results of neuropsychological testing (comparison with population norms versus player's own baseline test results) and potential problems of such testing in football. Finally, suggested recommendations for neuropsychological testing in football are given.
$\mathrm{T}$ he past two decades have seen a rapid increase in the use of neuropsychological testing in sports medicine, particularly in the management of concussive injury. The field has advanced to the point where neuropsychological testing is now recognised by many international sporting organisations and many practising sports medicine physicians as a critical component of the clinical management process following concussion. ${ }^{1-3}$

\section{BACKGROUND}

Traditionally the focus of neuropsychology has been on assessment of cognitive processes in order to anatomically localise structural brain injuries. ${ }^{4}$ For example, the French anatomist Paul Broca identified dysfluent speech production in two patients with damage to the posterior inferior frontal gyrus from bullet wounds. ${ }^{5}$ This science predates contemporary neuroimaging techniques which have largely replaced the patho-anatomical aspects of neuropsychology. However, the need to functionally assess patients with neurological disease and follow their progress over time has become more important in the modern era. This latter development arose largely from the work of the Russian psychologist, Alexandr Luria in the early twentieth century. Luria proposed that most behaviours were the result of complex functional systems in the brain and that lesions in any one structural component of these systems would cause a measurable disturbance in functional activity. ${ }^{6}$ The tools used by Luria and his colleagues consisted of a range of cognitive tests, from which responses could be observed and inferences drawn about an individual's brain function.

Over time, particularly in the USA, the application of statistical methods to define quantitative abnormalities in cognitive function has become prominent (psychometrics). Conversely, Luria's clinical-theoretical approach involves qualitative observation and subjective assessment of an individual's performance on cognitive tests.

\section{HISTORY OF NEUROPSYCHOLOGICAL TESTING IN SPORTS CONCUSSION}

From a clinical perspective, one of the major difficulties in the management of sports concussion has been the reliance on the subjective reporting of symptoms by athletes. The idea that neuropsychological tests could be used to provide an objective measure of cognitive recovery in the concussed athlete initially arose from work in severe traumatic brain injury. $^{7-9}$
Arising simultaneously in the USA and Australia, two distinct groups began groundbreaking research into the role of abbreviated neuropsychological assessment in sports concussion. Traditional approaches to cognitive assessment involving extensive testing by trained neuropsychologists were impractical for the evaluation of large groups of athletes.

In the USA, Barth and colleagues assessed concussed athletes using tests that had previously been shown to be sensitive in mild head trauma. ${ }^{10-12}$ The highpoint of this research resulted in the development of the Pittsburgh Steelers Test Battery which consisted of nine different tests evaluating a range of cognitive functions including verbal memory and delayed recall (Hopkins verbal learning tests), attention span and concentration (digit span test), motor speed and coordination (grooved pegboard test), and visual scanning, mental flexibility, and "throughput" (trail making test, symbol digit modalities). ${ }^{13}$ The overall time required for administration of these tests was approximately 45 minutes. Following this development, several major North American professional sporting organisations, such as the National Football League and the National Hockey League, commenced widespread concussion testing programmes at the elite level.

In Australian football, Maddocks and colleagues independently developed a screening tool for the diagnosis of concussion in sport as well as developing an abbreviated neuropsychological test battery that was sensitive to the cognitive deficits seen in concussion. ${ }^{14-17}$ They determined that the digit symbol substitution task (from the Wechsler Adult Intelligence Scale-Revised), paced auditory serial addition task, and choice reaction time were the most sensitive tools in the assessment of concussed players. The added advantage was that these tests were quick and easy to administer and were readily accepted in terms of face validity by subjects.

Over the past decade there has been increased interest in the use of computer administered tests of cognitive function. ${ }^{18-27}$ One of the main driving forces has been the explosion in advancement of computer technology, which has resulted in increasingly powerful and reliable portable technology. Although there are a number of practical and theoretical advantages of computerised testing over conventional paper and pencil neuropsychological testing it must be remembered that, despite their potential, computerised cognitive tests have not yet been validated for use in the follow up of sports related concussion. 
In addition, it must be remembered that the use of brief neuropsychological test batteries represents a screening approach to cognitive assessment. Although it facilitates baseline assessment of large numbers of athletes in the preseason, it does not allow for comprehensive evaluation of cognitive processes and thus may be limited with regard to sensitivity and specificity. ${ }^{21}{ }^{28}$ The corollary of this is that it is critical to have studies addressing the psychometric properties of the cognitive tests used in the management of sports concussion.

\section{MODERN APPROACH TO NEUROPSYCHOLOGICAL TESTING OF SPORTS CONCUSSION}

Despite the recommendation of expert consensus groups that neuropsychological testing is a key element of concussion management many key questions remain unresolved, such as:

- Which test or combination of tests is the most useful in the setting of sports concussion?

- How should the test results be interpreted?

- What factors impact upon test performance and how are these best controlled for?

\section{Which test is best?}

A different strategy of cognitive assessment is required when diagnosing concussion compared with when determining recovery to baseline performance. This difference reflects both the nature of the deficits encountered at each time point and the practicalities of assessment of athletes.

\section{Diagnosing concussion}

In practical terms, sports clinicians need a simple and valid tool that can be administered in an on-field situation to determine whether an athlete is concussed and hence should be removed from the field of play to be assessed more fully. From the work by Maddocks et al described above it was found that questions of recent and remote memory were the most sensitive measures separating concussed from nonconcussed athletes. These questions ("Maddocks' questions", see box below) were prospectively validated in a study of Australian football. ${ }^{14}$

In sports where athletes need to be assessed rapidly, often without removing them from the field, such as in football, these questions are an important tool fulfilling the basic requirements outlined above. Standard orientation questions, such as those pertaining to time, place, and person, are less discriminating in the sporting situation than questions on recently acquired memory. ${ }^{142}$ Other validated diagnostic tools included the Standardised Assessment of Concussion (SAC) which is a paper and pencil tool assessing orientation, concentration, and immediate and delayed memory. ${ }^{29}{ }^{30}$

\section{Maddocks' questions ${ }^{14}$}

- Which ground are we at?

- Which team are we playing today?

- Who is your opponent at present?

- Which half is it?

- How far into the quarter is it?

- Which side scored the last goal?

- Which team did we play last week?

- Did we win last week?
Although it has been validated in sports concussion, it is more time consuming than Maddocks' questions.

In addition to these two published and validated tools, a variety of other sideline assessment tools are there, most of which have not been prospectively validated and, in some cases, not widely published. These include:

- Sideline evaluation for concussion (Colorado Head Injury Foundation, Inc. $)^{31}$

- Management of concussion sports palm card (American Academy of Neurology and Brain Injury Association) ${ }^{32}$

- Sideline Concussion Check (Sports Medicine New Zealand Inc.) (unpublished)

- McGill Abbreviated Concussion Evaluation (unpublished)

- National Hockey League Physician Evaluation Form (unpublished)

- The UK Jockey Club Assessment of Concussion ${ }^{33}$

All these tools were recently combined into a single sideline assessment tool developed by the Prague consensus group. ${ }^{34}$ This tool, called the "Sport Concussion Assessment Tool" (SCAT), was developed through a process of collaboration and iterative review, and evaluated for face and content validity on the basis of scientific literature ${ }^{3}$ and clinical experience of the authors. It will need to be validated in prospective studies before it can be widely implemented.

It is important to note that none of these tools will necessarily exclude a more significant intracranial injury which may masquerade as a concussion in the early stages. Hence, these tools are not a substitute for a formal medical assessment.

\section{Serial follow up and return to play}

Whereas assessment of memory is the critical aspect of neuropsychological testing in the setting of concussion diagnosis, the assessment of recovery mandates a different test strategy. This is based not only on the known neuropsychological deficits that exist following concussion but also on the different time frame available for follow up assessment that allows a more detailed assessment to take place.

In addition to the clinical symptoms of concussion, a range of neuropsychological deficits may be observed in the recovery phase following a concussive injury. These deficits are typically subtle and mild and include:

- reduced planning and ability to switch mental set t $^{35-38}$

- impaired memory and learning ${ }^{99-41}$

- reduced attention and ability to process informa$\operatorname{tion}^{17264243}$

- slowed reaction times and increased variability in response. 2227445

Isolated reports have suggested that impairments may be evident on tasks involving visuospatial constructional ability, language, and sensorimotor function. ${ }^{35}{ }^{36}$ One area of concern that has not been studied in detail is the proposal that following recovery, deficits may still be evident when assessed under conditions of physiological stress. ${ }^{46}$ The corollary of this is that any form of neuropsychological testing needs to be sensitive for the changes observed following concussion and be specific for these deficits.

It must be emphasised however, that neuropsychological assessment should not be the sole basis of a return to play decision but rather be seen as an aid to the clinical decision making. In general terms, neuropsychological testing should not be routinely done while the athlete is symptomatic since it adds little to decisions regarding return to play and it may 


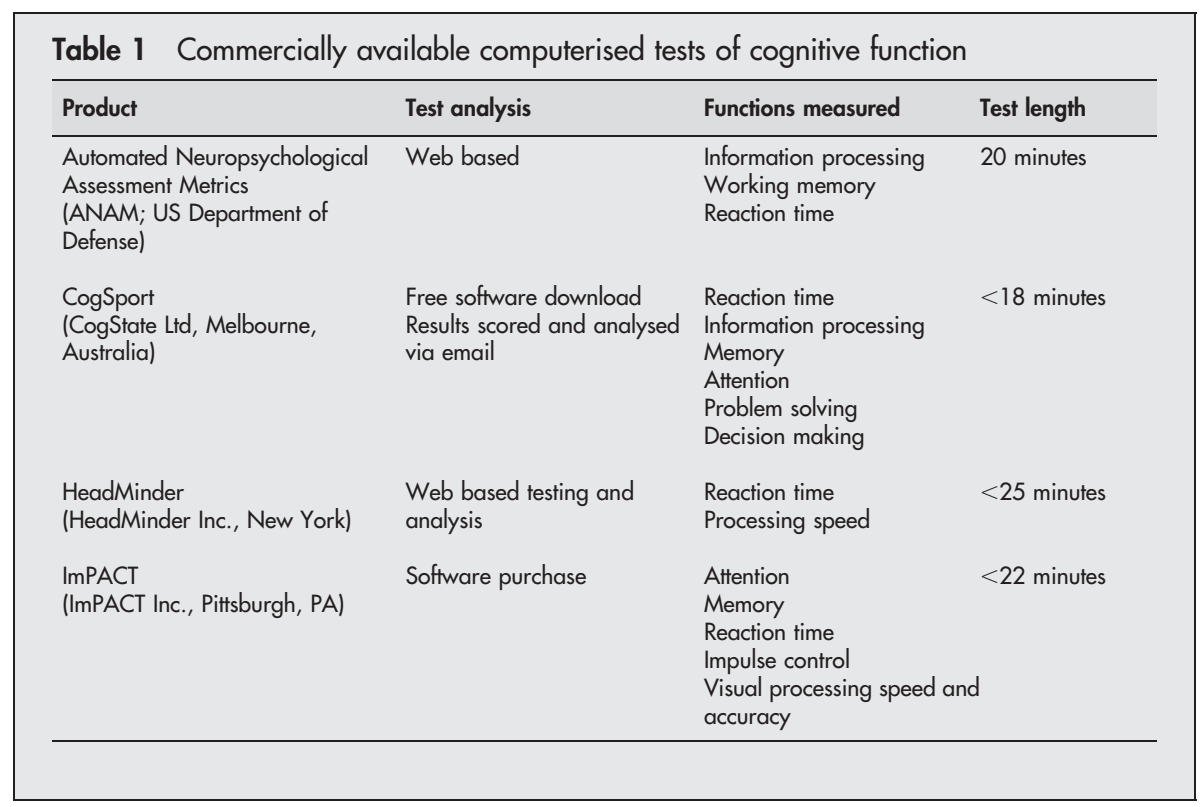

contaminate the testing process by allowing for practise effects to confound the results. The recommended consensus strategy is to wait for the resolution of clinical symptoms (both at rest and with provocative exercise challenge) and then to use the neuropsychological testing as the final step in the return to play strategy. ${ }^{34}$

In terms of determining which test is most useful in the assessment of sports concussion, the choice lies between traditional paper and pencil tests and computerised test platforms. Whichever strategy is chosen requires that the relevant range of cognitive abnormalities outlined above is detected by the tests used.

The major problem is that conventional paper and pencil neuropsychological tests suffer from psychometric confounds that make them less than ideal for such serial use in sport, including a lack of equivalent alternative forms, poor testretest reliability, and susceptibility to interrater biases and practise effects. ${ }^{19}$ Furthermore, the limited availability of neuropsychologists to administer and interpret the test results makes the widespread application of such testing problematical.

Computerised test platforms allow for the widespread use of neuropsychological testing in the sporting situation. The benefits and disadvantages of the different commercially available test platforms have been reviewed in detail elsewhere and are summarised below and in table $1 .{ }^{19}$ These products are practical and in some cases do not require a neuropsychologist to interpret or administer the test. ${ }^{47}$

The potential advantages of computerised testing over conventional paper and pencil neuropsychological testing include:

- standardisation of stimulus presentation (that is, stimulus presentation and contingency onset are controlled by the software thereby minimising any inter-assessor or intraassessor variability/unreliability)

- short administration time/rapid testing

- sensitivity-accurate in detecting deficits well below the measurement capabilities of traditional clinical neuropsychological tests (that is, detect deficits in reaction time in the order of 30-110 ms)

- presentation of multiple and equivalent alternative forms of a test within a relatively brief period of time (useful for monitoring cognitive function over time and minimising the effects of practise)
- accurate analysis of performance stability/variability ${ }^{26} 48$

- computerised analysis (sensitive to small changes/subtle differences)

- centralised data storage, analysis, and reporting

- potential for internet based delivery (practical/convenient).

\section{How do we interpret the results?}

Cognitive testing in sports concussion may be interpreted by comparing the results either with population norms or with the individual's own baseline (or pre-injury) performance.

\section{Comparison with group norms}

In general, most individuals referred for neuropsychological testing, whether in a sporting or hospital setting, are unlikely to have pre-existing baseline testing performed. In these patients, the application of a battery of neuropsychological tests allows detection of gross cognitive deficits when compared with population norms. There are a number of problems with using this technique in the assessment of athletes following sports concussion:

- Deficits observed following sports concussion are often subtle.

- A large range of factors impact on a player's test performance, thus any deficits observed in relation to population norms must be interpreted with caution.

- Many of the common tests used for the assessment of sports concussion have a wide range of normal results.

- There are limited normal datasets for specific population groups of interest (in particular, young athletes and females).

\section{"Back to baseline" model}

The most effective use of neuropsychological testing in sport involves a baseline assessment of the athlete's pre-injury level of cognitive functioning. This allows direct comparison with the post-concussive test results and subsequently controls for many of the individual athlete factors that can impact on test performance. ${ }^{21} 334950$ When using individual baseline tests for comparison it is not enough to simply subtract the postconcussion score from the baseline, as this fails to account for 


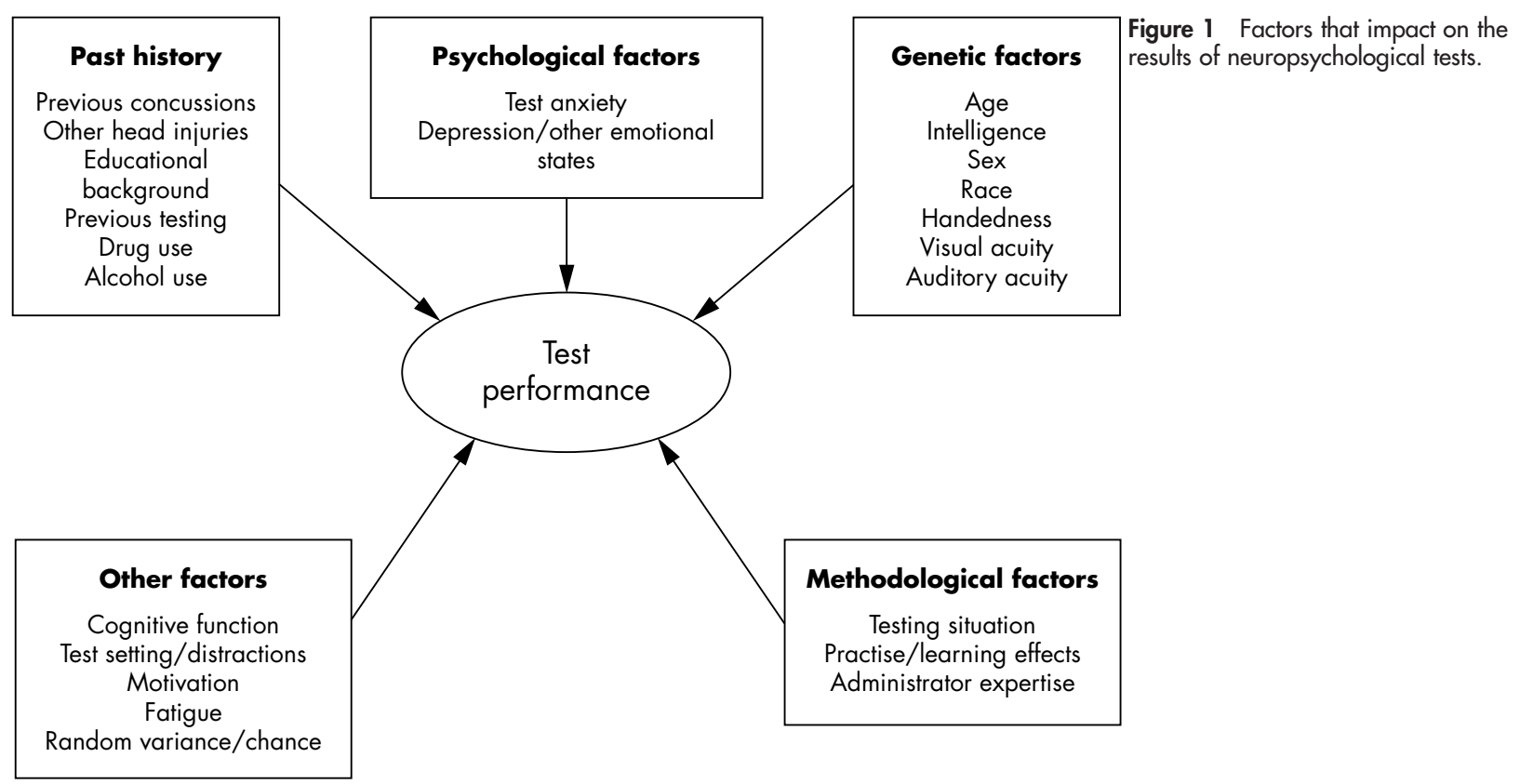

the many factors that may confound interpretation of serially acquired cognitive test scores, such as the practise effect. ${ }^{19}$

A variety of approaches have been used to differentiate "true" changes from changes caused by these confounding factors. ${ }^{25} 50-55$ The two commonest are the reliable change index, which takes into account the test-retest reliability of the measure, and standardised regression based measures, which use a more sophisticated statistical approach to account for potential sources of test-retest bias. With the current computerised test platforms, these indices are calculated automatically and an interpretive report summary provided.

\section{WHAT ARE THE POTENTIAL PROBLEMS OF NEUROPSYCHOLOGICAL TESTING?}

A range of neurological and non-neurological factors can significantly impact on an individual's performance on cognitive tests, both before and after sports concussion. ${ }^{28} 55$ These are summarised in fig 1 . Whereas one of the important interpretive aspects of traditional neuropsychology has been to determine the extent by which such factors may influence test outcomes, the newer computerised test platforms largely deal with most of, if not all, these statistical concerns leaving the clinician to interpret the findings in the light of the clinical situation.

It is important to note that there may be interaction between any of these factors and that test performance may also be affected by chance or random variance. Thus a major challenge in interpretation of the test result lies in differentiating the effects of concussion from the influence of other potential confounding factors. This is facilitated by the use of baseline testing.

\section{NEUROPSYCHOLOGICAL TESTING IN FOOTBALL}

Most of the recent studies on head injuries in football are descriptive in nature, highlighting the risk of acute injury to players. ${ }^{56-67}$ Only limited data exist regarding neuropsychological changes in this setting and the majority of published papers relate to concern about putative long term effects of concussive injury and/or heading the ball. ${ }^{37}$ 63 68-74

Whether repeated subconcussive trauma, such as might be seen in "heading" the ball, causes cumulative neurological injury remains speculative Although this idea was suggested by early retrospective studies, more recent studies have failed to provide support for it. ${ }^{71}$ In series of retrospective studies, involving retired Scandinavian football players, cognitive deficits were noted. ${ }^{75}{ }^{76}$ The results of these studies are flawed because of significant methodological problems. Problems include the lack of pre-injury data, selection bias, failure to control for acute head injuries, lack of observer blinding, and inadequate control subjects. The authors concluded that the deficits noted in the former football players were explained by repetitive trauma such as heading the ball, however, the pattern of deficits is equally consistent with alcohol related brain impairment, a confounding variable that was not controlled for.

Matser and colleagues from the Netherlands have also implicated both concussive injury and heading as a cause of neuropsychological impairment in both amateur and professional football players. ${ }^{37} 70{ }^{77}$ Reanalysis of the data from these papers, however, suggests that purposeful heading may not be a risk factor for cognitive impairment. ${ }^{69}$ Other prospective studies in football have failed to find any evidence of chronic cognitive impairment in players using clinical examination, neuroimaging, or neuropsychological testing. ${ }^{65} 7879$

It seems unlikely that subconcussive impacts such as seen in head to ball contact will cause chronic neurological injury, and although head to head contact may cause concussive injury, it is both uncommon and unlikely to result in cumulative brain injury. It has been speculated from other sports that particular genotypes may place athletes at heightened risk in association with head trauma although this remains to be validated in other studies. ${ }^{80}$

\section{Recommendations for neuropsychological testing in football}

The broad recommendations for the overall management of concussive injuries have been recently summarised in the Prague consensus document. ${ }^{34}$ With regard to neuropsychological testing the following points can be suggested:

- Diagnosis of concussion or suspected concussion should be made using either Maddocks' questions or the relevant section of the SCAT form. This assessment can be made by medical or non-medical personnel. 
- All players diagnosed as having had a concussion require a medical assessment.

- Return to play decisions require a cognitive assessment once a player is asymptomatic both at rest and following exercise. At the elite level, it is recommended that one of the computerised test platforms is used with a "back to baseline" strategy of documenting recovery. At the nonelite level, return to play should follow the stepwise process outlined in the Prague consensus statement.

\section{CONCLUSION}

Neuropsychological test batteries have been added to the management of sports concussion to provide objective information about both diagnosis and recovery following injury. Given the wide range of factors that can influence test performance, the most accurate interpretations can be made when an individual's results are compared with their own baseline. At present a variety of traditional paper and pencil protocols and newer computerised test batteries exist. An understanding of the limitations and interpretation of such tests will assist the clinician in the use of these tests as an adjunct to clinical assessment.

\section{Authors' affiliations}

P McCrory, M Makdissi, Centre for Health, Exercise and Sports Medicine, University of Melbourne, Melbourne, Victoria, Australia G Davis, Cabrini Medical Centre, Malvern, Victoria, Australia A Collie, CogState Ltd., Melbourne, Victoria, Australia

Competing interests: A Collie is employed by CogSport, a commercial computerised concussion management software company. None of the other authors has a declared conflict of interest.

\section{REFERENCES}

1 Aubry M, Cantu R, Dvorak J, et al. Summary and agreement statement of the first International Conference on Concussion in Sport, Vienna 2001. Br J Sports Med 2002;36:6-10

2 McCrory P, Johnston K, Meeuwisse W, et al. Summary and Agreement Statement on the Second International Conference on Concussion in Sport, Prague 2004. Phys Sportsmed 2005;33:29-36.

3 Johnston K, McCrory P, Mohtadi N, et al. Evidence based review of sportrelated concussion-clinical science. Clin J Sport Med 2001;11:150-60.

4 Walsh KMA. Understanding brain damage: A primer of neuropsychological evaluation, 2nd edn. Edinburgh: Churchill Livingstone, 1991

5 McCrory P, Berkovic S. Concussion: Historical development of clinical and pathophysiological concepts and misconceptions. Neurology 2001; 57:2283-9.

6 Luria AR. Higher cortical functions in man, 2nd edn. New York: Basic Books Inc, 1962.

7 Gronwall D. Performance changes during recovery from closed head injury Proc Aust Assoc Neurol 1976;13:143-7.

8 Gronwall D. Cumulative and persisting effects of concussion on attention and cognition. In: Levin H, Eisenberg H, Benton A, eds. Mild Head Injury. New York: Oxford University Press, 1989:153-62.

9 Gronwall D, Wrighson P. Memory and information processing capacity after closed head injury. I Neurol Neurosurg Psychiatry 1981;44:889-95.

10 Barth JT, Alves WM, Ryan TV, et al. Mild head injury in sports: neuropsychological sequelae and recovery of function. In: Levin HS, Eisenberg HM, Benton AL, eds. Mild Head Injury. New York: Oxford University Press, 1989:257-75.

11 Barth JT, Freeman JR, Winters JE. Management of sports-related concussions. Dent Clin North Am 2000;44:67-83.

12 Barth JT, Macciocchi SN, Giordani B, et al. Neuropsychological sequelae of minor head injury. Neurosurgery 1983;13:529-33.

13 McKeag D. Mild brain trauma: a team physician's prospective. In: American Medical Society for Sports Medicine: Invited symposium on mild brain injury. Cincinnati, Ohio, 1996:1-2.

14 Maddocks DL, Dicker GD, Saling MM. The assessment of orientation following concussion in athletes. Clin J Sport Med 1995;5:32-5.

15 Maddocks D, Saling M. Neuropsychological sequelae following concussion in Australian Rules footballers. J Clin Exp Neuropsychol 1991;13:439-41.

16 Maddocks D, Saling M. Neuropsychological deficits following concussion. Brain Inj 1996; 10:99-103.

17 Maddocks DL, Saling MM, Dicker GD. A note on the normative data for a test sensitive to concussion in Australian Rules footballers. Aust Psychol 1995:30:125-7.

18 Bleiberg J, Halpern E, Reeves D, et al. Future directions for the neuropsychological assessment of sports concussion. J Head Trauma Rehabil 1998;13:36-45.
19 Collie A, Darby D, Maruff P. Computerised cognitive assessment of athletes with sports-related head injury. Br J Sports Med 2001;35:297-302.

20 Erlanger DSE, Barth J, Almquist J, et al. Monitoring resolution of post concussion symptoms in athletes: preliminary results of a web-based neuropsychological test protocol. J Athl Train 2001;36:280-7.

21 Lovell MR, Collins MW. Neuropsychological assessment of the college football player. J Head Trauma Rehabil 1998;13:9-26.

22 Warden D, Bleiberg J, Cameron K, et al. Persistent prolongation of simple reaction time in sports concussion. Neurology 2001:57:524-6.

23 Daniel J, Olesniewicz M, Reeves D, et al. Repeated measures of cognitive processing efficiency in adolescent athletes: implications for monitoring recovery from concussion. Neuropsychiatry Neuropsychol Behav Neurol 1999:12:167-9.

24 Echemendia R, Cantu R. Return to play following sports-related mild traumatic brain injury: the role for neuropsychology. Appl Neuropsychol 2003;10:48-55.

25 Erlanger D, Feldman D, Kutner K, et al. Development and validation of a webbased neuropsychological test protocol for sports-related return-to-play decision-making. Arch Clin Neuropsychol 2003;18:293-316.

26 Stuss D, Stethem L, Hugenholz H, et al. Reaction time after head injury: fatigue, divided and focussed attention and consistency of performance. J Neurol Neurosurg Psychiatry 1989;52:742-8.

27 Collins M, Field M, Lovell $M$, et al. Relationship between post concussion headache and neuropsychological test performance in high school athletes. Am J Sports Med 2003;31:168-73.

28 Grindel S, Lovell M, Collins M. The assessment of sport-related concussion: the evidence behind neuropsychological testing and management. Clin J Sport Med 2001;11:134-44.

29 McCrea M, Kelly J, Randolph C, et al. Standardised assessment of concussion (SAC): On site mental status evaluation of the athlete. J Head Trauma Rehab 1998;13:27-36.

30 McCrea M. Standardized mental status testing on the sideline after sportrelated concussion. J Athl Train 2001;36:274-9.

31 Society CM. Report of the sports medicine committee: guidelines for the management of concussions in sport (revised). Denver: Colorado Medical Society, 1991.

32 Kelly J, Rosenberg J. Diagnosis and management of concussion in sports Neurology 1997;48:575-80.

33 Turner M. Concussion and head injuries in sport. In: Turner M, ed. The Jockey Club Conference on head injury in sport. London: The Jockey Club of England, 1998.

34 McCrory P, Johnston K, Meeuwisse W, et al. Summary and agreement statement of the second International Conference on Concussion in Sport, Prague 2004. Br J Sports Med 2005;39(suppl 1):i78-i86.

35 Barth J, Freeman J, Broshek D, Varney R. Acceleration-deceleration sportrelated concussion: the gravity of it all. J Athl Train 2001;36:253-6.

36 Leninger BE, Gramling SE, Farrell AD, et al. Neuropsychological deficits in symptomatic minor head injury patients after concussion and mild head injury. J Neurol Neurosurg Psychiatry 1990;53:293-6.

37 Matser EJ, Kessels AG, Lezak MD, et al. Neuropsychological impairment in amateur soccer players [see comments]. JAMA 1999;282:971-3.

38 Rimel RW, Giordani B, Barth JT, et al. Moderate head injury: completing the clinical spectrum of brain trauma. Neurosurgery 1982;11:344-51.

39 Dikmen S, Mclean A, Temkin N. Neuropsychological and psychological consequences of minor head injury. J Neurol Neurosurg Psychiatry 1986;49:1227-32.

40 Gusckiewicz K, Ross SE, Marshall SW. Postural stability and neuropsychological deficits after concussion in collegiate athletes. J Athl Train $2001 ; 36: 263-73$

41 Lovell MR, Collins MW, Iverson GL, et al. Recovery from mild concussion in high school athletes. J Neurosurg 2003;98:296-301.

42 Maddocks DL, Saling M, Dicker GD. Normative data for a test sensitive to concussion in Australian Rules footballers. Information Processing In Footballers 1993: 1-12.

43 Stuss DT, Stethem LL, Hugenholz $\mathrm{H}$, et al. Traumatic brain injury: a comparison of three clinical tests and analysis of recovery. Clin Neuropsychol 1989;3:145-56.

44 Makdissi M, Collie A, Maruff $P$, et al. Computerised cognitive assessment of concussed Australian Rules footballers. Br J Sports Med 2001;35:354-60.

45 Moriarity J, Collie A, Olson D, et al. A prospective controlled study of cognitive function during an amateur boxing tournament. Neurology 2004;62: 1497-502

46 Ewing R, McCarthy D, Gronwall D, et al. Persisting effects of minor head injury observable during hypoxic stress. J Clin Neuropsychiatry 1980;2:147-55.

47 Collie A, Maruff P. Computerised neuropsychological testing. Br J Sports Med 2003;37:2-3.

48 Bleiberg J. Consistency of within-day and across-day performance after mild brain injury. Neuropsychiatry Neuropsychol Behav Neurol 1997;10:247-53.

49 Hinton-Bayre AD, Geffen G, McFarland K. Mild head injury and speed of information processing: a prospective study of professional rugby league players. J Clin Exp Neuropsychol 1997;19:275-89.

50 Hinton-Bayre AD, Geffen GM, Geffen LB, et al. Concussion in contact sports: reliable change indices of impairment and recovery. J Clin Exp Neuropsychol 1999;21:70-86.

51 Barr WB. Methodologic issues in neuropsychological testing. J Athl Train 2001;36:297-302.

52 Barr W. Neuropsychological testing of high school athletes-preliminary norms and test-retest indices. Arch Clin Neuropsychol 2003;18:91-101. 
53 Collie A, Maruff P, Darby DG, et al. The effects of practice on the cognitive test performance of neurologically normal individuals assessed at brief test-retest intervals. J Int Neuropsychol Soc 2003;9:419-28.

54 Collie A, Maruff P, Makdissi M, et al. Cogsport: Reliability and correlation with conventional cognitive tests used in post concussion medical evaluations. Clin J Sport Med 2003;13:28-33

55 Collie A, Maruff P, Makdissi M, et al. Statistical procedures for determining the extent of cognitive change following concussion. Br J Sports Med 2004;38:273-8.

56 Barnes BC, Cooper L, Kirkendall DT, et al. Concussion history in elite male and female soccer players. Am J Sports Med 1998;26:433-8.

57 Boden B, Kirkendall D, Garrett W. Concussion incidence in elite college soccer players. Am J Sports Med 1998;26:238-41.

58 Bruce DA, Schut L, Sutton LN. Brain and cervical spine injuries occurring during organized sports activities in children and adolescents. Prim Care 1984;11:175-94.

59 Chan KM, Fu F, Leung L. Sports injuries survey on university students in Hong Kong. Br J Sports Med 1984; 18:195-202.

60 Dailey SW, Barsan WG. Head injuries in soccer. Phys Sportsmed 1992;20:79-85.

61 Faulkner C, School M. Injury rates in non-professional football and soccer. Aust J Physiother 1985;31:242-3.

62 Fields KB. Head injuries in soccer. Phys Sportsmed 1989;17:69-73.

63 Green GA, Jordan SE. Are brain injuries a significant problem in soccer? Clin Sports Med 1998; 17:795-809, viii.

64 Inklaar H. Soccer injuries I: incidence and severity. Sports Med 1994; 18:55-73.

65 Jordan BD. Acute and chronic brain injury in United States national team soccer players. Am J Sports Med 1996;24:704-5.

66 Juma AH. Outline of sport injuries in the V World Youth Championship for FIFA Cup in Saudi Arabia. Indian J Med Sci 1998;52:433-7.
67 Kujala UM, Taimela S, Antti-Poika I, et al. Acute injuries in soccer, ice hockey, volleyball, basketball, judo, and karate: analysis of national registry data. BMJ 1995;311:1465-8.

68 Abreau F, Templer DI, Schuyler BA, et al. Neuropsychological assessment of soccer players. Neuropsychology 1990:4:175-81.

69 Kirkendall DT, Garrett WE. Heading in soccer: integral skill or grounds for cognitive dysfunction? J Athl Train 2001;36:328-33.

70 Matser JT, Kessels AGH, Jordan BD, et al. Chronic traumatic brain injury in professional soccer players. Neurology 1998;51:791-6.

71 McCrory P. Brain injury and heading in soccer. BMJ 2003;327:351-2.

72 McGrath A, Ozanne-Smith J. Heading injuries out of soccer: a review of the literature. Victoria, Australia: Accident Research Centre, Monash University, 1997.

73 Health Council of the Netherlands. Brain damage in boxers and soccer players., 2003:1-80.

74 Tysvaer A, Lochen E. Soccer injuries to the brain: a neuropsychological study of former soccer players. Am J Sports Med 1991;19:56-60.

75 Tysvaer A, Storli O, Bachen N. Soccer injuries to the brain: a neurologic and encephalographic study of former players. Acta Neurol Scand 1989;80:151-6.

76 Tysvaer AT. Head and neck injuries in soccer the impact of minor head trauma. Sports Med 1992;14:200-13.

77 Matser JT, Kessels AGH, Lezak MD, et al. A dose-response relation of headers and concussions with cognitive impairment in professional soccer players. J Clin Exp Neuropsychol $2001 ; 23: 770-4$.

78 Putukian M, Echemendia R. Managing successive minor head injuries. Which tests guide return to play? Phys Sportsmed 1996;24:25-38.

79 Guskiewicz KM, McCrea M, Marshall SW, et al. Cumulative effects associated with recurrent concussion in collegiate football players. JAMA 2003;290:2549-55.

80 Jordan B. Genetic susceptibility to brain injury in sports: a role for genetic testing in athletes? Phys Sportsmed 1998;26:25-6. 